\title{
Practicable Scheme to Generate the Three Photon Entangled State with Linear Optical Elements
}

\author{
Siriporn Saiburee and Wanchai Pijitrojana \\ Department of Electrical and Computer Engineering, Thammasat University \\ 9 Moo 18 , Khlong Luang, Pathumthani, Thailand, 12121 \\ siriporn saiburee@nectec.or.th,pwanchai@engr.tu.ac.th
}

\begin{abstract}
This paper proposed practicable scheme for generation of the three photon entangled states without any nonlinear optical process. The three-photon entanglement can be achieved by using linear optical elements with post selection based on the photons interferometry. The success probability of proposed method is $3 / 16$, higher than the previous methods using the linear optical system.
\end{abstract}

\section{Keywords - Entangled photon, Photon interference, Optical element, Beam splitter}

\section{INTRODUCTION}

Entanglement is a non-local more characteristic with higher correlation than classical systems. The entangled states can be used for quantum information and communication such as quantum key distribution, teleportation and quantum computing. These applications require a large number of entangled state from primary resource. There are various methods to generate entangled state of two or more photon such as atomic, optical nonlinear crystal, optical fiber, semiconductors and optical linear elements. Most recently, the semiconductor and atomic are potential resources for the generation of entangled photon in the future. However, these technique are still very difficult to generate and maintain the entangled photon state such fragile environments. Parametric down conversion (PDC) in optical nonlinear crystals has been the most popular and powerful method of obtaining entangled photons. The pump light is converted into two lights (signal and idler) in a crystal with optical non-linearity. For example, the generation of polarization entangled photons using PDC with post selection procedure based on the photons interferometry as in [1]-[9]. Next, Kwiat et al. proposed the polarization entanglement was generated by a PDC crystal without the use of post selection as in [10]-[11]. Another method applying PDC to place nonlinear crystal in an interferometer such as Mach Zehnder and Sagnac in [12]-[22]. In addition, the optical linear elements is alternative technology for using to generate entangled photon which the capital cost of research lower another method. For instance, in [23] is proposed a scheme to produce an entangled four photon with linear optical elements which the entangled source is generated the initial state for system. The scheme for create four photon W states has been proposed in [24]. Next, in [25] was shown that the Greenberger-Horne-Zeilinger (GHZ) state of $\mathrm{N}$ photon was produced by simple linear optical elements. The optical gate for the generating to GHZ and $\mathrm{W}$ states with linear optical elements has been proposed by paper in [26]-[27]. However, there are some methods refers the physical of nonlinear crystals to an initially entangled source. For example, the entanglement source was used by paper in [23], [26] to create four photon entangled states.

This paper emphasizes to enhance generating for three entangled photon by using the linear optical elements with post selection based on the photons interferometry. Hence, the technical challenge of method is the generation of entangled photons without the PDC in nonlinear optical crystals. In addition, practicable scheme can be achieved in applications of quantum key distribution, teleportation and quantum computation.

\section{ProPOSED METHOD}

This paper proposed feasible scheme for generation of three photon entangled state as shown in Figure1. The method can be achieved by using linear optical elements with post selection based on the photons interferometry. The scheme is composed of single photon source, two beam splitters (50:50) and single photon detectors. The specific input state was prepared by single photon sources with one photon and two photons which both are injected into first beam splitter (BS1) from mode 1 and 2 respectively. There are four possible after BS1: (a) all photons are appears in mode 4, (b) one photon and two photons are appears in mode 3 and 4, (c) two photons and one photon are appears in mode 3 and 4, (d) all photons are appears in mode 3 respectively. One output of BS1 is divided into two modes of second beam splitter (BS2). Finally, the generating of three-photon entangled photon is successful when each of spatial mode has one photon with perfect post selection.

In consideration, the input states are defined by $|H\rangle_{1}$ and $|H V\rangle_{2}$ which the number in the subscript is the spatial mode of input state. The action of BS on horizontal polarization $(\mathrm{H})$ can be described by the creation operators as

$$
\hat{a}_{1 \mathrm{H}}^{\dagger}=\frac{1}{\sqrt{2}}\left(\hat{a}_{3 \mathrm{H}}^{\dagger}+\hat{a}_{4 \mathrm{H}}^{\dagger}\right), \hat{a}_{2 \mathrm{H}}^{\dagger}=\frac{1}{\sqrt{2}}\left(\hat{a}_{3 \mathrm{H}}^{\dagger}-\hat{a}_{4 \mathrm{H}}^{\dagger}\right)
$$




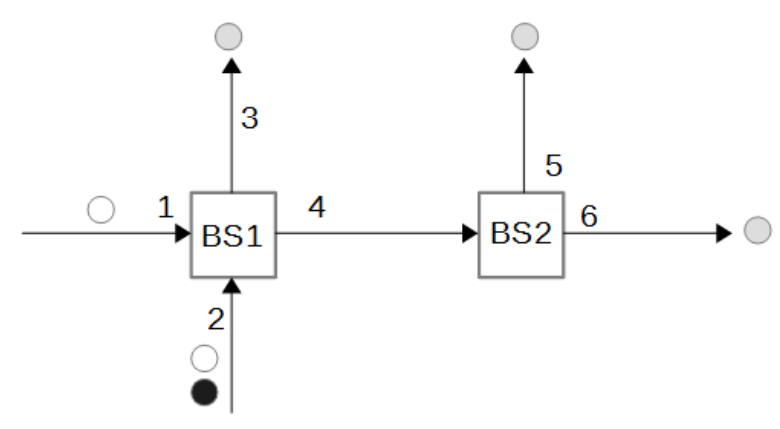

Figure 1. The schematic diagram of entangled three photon generation : one photon in the polarization state from mode 1 and two photons in the polarization state are injected into the BS1.

Similarly, the action of BS on vertical polarization (V) was denoted by

$$
\hat{a}_{1 \mathrm{~V}}^{\dagger}=\frac{1}{\sqrt{2}}\left(\hat{a}_{3 \mathrm{~V}}^{\dagger}+\hat{a}_{4 \mathrm{~V}}^{\dagger}\right), \quad \hat{a}_{2 \mathrm{~V}}^{\dagger}=\frac{1}{\sqrt{2}}\left(\hat{a}_{3 \mathrm{~V}}^{\dagger}-\hat{a}_{4 \mathrm{~V}}^{\dagger}\right) .
$$

Where $\hat{a}_{j H}^{\dagger}$ and $\hat{a}_{j V}^{\dagger}$ are the photon creation operators in $\mathrm{H}$ and $\mathrm{V}$ polarization for mode $\mathrm{j}$. Therefore, the output states after BS1 is according to

$$
\begin{aligned}
\left|1_{H}\right\rangle_{1}\left|1_{H} 1_{V}\right\rangle_{2}= & \frac{1}{\sqrt{2}}\left(\hat{a}_{1 \mathrm{H}}^{\dagger} \hat{a}_{2 \mathrm{H}}^{\dagger} \hat{a}_{2 \mathrm{~V}}^{\dagger}\right)|00\rangle_{3,4} \\
= & \frac{1}{2 \sqrt{2}}\left(\hat{a}_{3 \mathrm{H}}^{\dagger}+\hat{a}_{4 \mathrm{H}}^{\dagger}\right)\left(\hat{a}_{3 \mathrm{H}}^{\dagger}-\hat{a}_{4 \mathrm{H}}^{\dagger}\right)\left(\hat{a}_{3 \mathrm{~V}}^{\dagger}-\hat{a}_{4 \mathrm{~V}}^{\dagger}\right)|00\rangle_{3,4} \\
= & \frac{1}{2 \sqrt{2}}\left|2_{H} 1_{V}, 0\right\rangle_{3,4}-\frac{1}{2 \sqrt{2}}\left|2_{H}, 1_{V}\right\rangle_{3,4}+ \\
& \frac{1}{2 \sqrt{2}}\left|0,2_{H} 1_{V}\right\rangle_{3,4}-\frac{1}{2 \sqrt{2}}\left|1_{V}, 2_{H}\right\rangle_{3,4}+ \\
& \frac{1}{2}\left|1_{H} 1_{V}, 1_{H}\right\rangle_{3,4}+\frac{1}{2}\left|1_{H}, 1_{H} 1_{V}\right\rangle_{3,4}
\end{aligned}
$$

The method of generation is successful when each of the output mode 3, 5 and 6 has one photon. Therefore, there are two terms which are useful for the generation of entangled three photon as

$$
\frac{1}{2 \sqrt{2}}\left|1_{V}, 2_{H}\right\rangle_{3,4} \text { and } \frac{1}{2}\left|1_{H}, 1_{H} 1_{V}\right\rangle_{3,4} .
$$

The states $\left|2_{H}\right\rangle_{4}$ and $\left|1_{H} 1_{V}\right\rangle_{4}$ are injected into the BS2 which the output state can be given by

$$
\begin{aligned}
\left|2_{H}\right\rangle_{4}= & \frac{1}{2}\left|2_{H}, 0\right\rangle_{5,6}+\frac{1}{\sqrt{2}}\left|1_{H}, 1_{H}\right\rangle_{5,6}+\frac{1}{2}\left|0,2_{H}\right\rangle_{5,6} \\
\left|1_{H} 1_{V}\right\rangle_{4}= & \frac{1}{2}\left|1_{H} 1_{V}, 0\right\rangle_{5,6}+\frac{1}{2}\left|1_{H}, 1_{V}\right\rangle_{5,6}+\frac{1}{2}\left|1_{V}, 1_{H}\right\rangle_{5,6}+ \\
& \frac{1}{2}\left|0,1_{H} 1_{V}\right\rangle_{5,6}
\end{aligned}
$$

After the BS2, one photon of the output mode 5 and 6 are useful for generation of entangled three photon. Therefore, the output state becomes the entangled three photon can be achieved by post selection as follows

$$
\left|1_{H}\right\rangle_{1}\left|1_{H} 1_{V}\right\rangle_{2}=\frac{1}{4}\left(|V H H\rangle_{356}+|H V H\rangle_{356}+|H H V\rangle_{356}\right)
$$

Similarly, if one photon in the polarization state $|H\rangle_{1}$ and two photons in the polarization state $|V V\rangle_{2}$ are injected into BS1. The output state can be described by

$$
\begin{aligned}
\left|1_{H}\right\rangle_{1}\left|1_{V} 1_{V}\right\rangle_{2}= & \frac{1}{2 \sqrt{2}}\left|1_{H} 2_{V}, 0\right\rangle_{3,4}+\frac{1}{2}\left|1_{H} 1_{V}, 1_{V}\right\rangle_{3,4}+ \\
& \frac{1}{2 \sqrt{2}}\left|1_{H}, 2_{V}\right\rangle_{3,4}+\frac{1}{2 \sqrt{2}}\left|0,1_{H} 2_{V}\right\rangle_{3,4}+ \\
& \frac{1}{2 \sqrt{2}}\left|2_{V}, 1_{H}\right\rangle_{3,4}-\frac{1}{2}\left|1_{V}, 1_{H} 1_{V}\right\rangle_{3,4}
\end{aligned}
$$

The method of generation is successful when choose the events that there are one photon in mode 3, 5 and 6 . Two terms of output state are useful for the generation of entangled three photon as $\frac{1}{2 \sqrt{2}}\left|1_{H}, 2_{V}\right\rangle_{3,4}$ and $\frac{1}{2}\left|1_{V}, 1_{H} 1_{V}\right\rangle_{3,4}$.

The states $\left|2_{V}\right\rangle_{4}$ and $\left|1_{H} 1_{V}\right\rangle_{4}$ are injected into the BS2 which the output state can be given by

$$
\begin{aligned}
\left|2_{V}\right\rangle_{4}= & \frac{1}{2}\left|2_{V}, 0\right\rangle_{5,6}+\frac{1}{\sqrt{2}}\left|1_{V}, 1_{V}\right\rangle_{5,6}+\frac{1}{2}\left|0,2_{V}\right\rangle_{5,6} \\
\left|1_{H} 1_{V}\right\rangle_{4}= & \frac{1}{2}\left|1_{H} 1_{V}, 0\right\rangle_{5,6}+\frac{1}{2}\left|1_{H}, 1_{V}\right\rangle_{5,6}+\frac{1}{2}\left|1_{V}, 1_{H}\right\rangle_{5,6}+ \\
& \frac{1}{2}\left|0,1_{H} 1_{V}\right\rangle_{5,6}
\end{aligned}
$$

Consequently, the output state becomes the entangled three photon can be achieved by post selection as follows

$$
\left|1_{H}\right\rangle_{1}\left|1_{V} 1_{V}\right\rangle_{2}=\frac{1}{4}\left(|H V V\rangle_{356}+|V V H\rangle_{356}+|V H V\rangle_{356}\right)
$$

In addition, one photon in the polarization state $|V\rangle_{1}$ and two photons in the polarization state $|H H\rangle_{2}$ are input for BS1. The output state can be described by

$$
\begin{aligned}
\left|1_{V}\right\rangle_{1}\left|1_{H} 1_{H}\right\rangle_{2}= & \frac{1}{2 \sqrt{2}}\left|1_{V} 2_{H}, 0\right\rangle_{3,4}-\frac{1}{2}\left|1_{V} 1_{H}, 1_{H}\right\rangle_{3,4}+ \\
& \frac{1}{2 \sqrt{2}}\left|1_{V}, 2_{H}\right\rangle_{3,4}-\frac{1}{2}\left|1_{H}, 1_{V} 2_{H}\right\rangle_{3,4}+ \\
& \frac{1}{2 \sqrt{2}}\left|0,1_{V} 2_{H}\right\rangle_{3,4}+\frac{1}{2 \sqrt{2}}\left|2_{H}, 1_{V}\right\rangle_{3,4}
\end{aligned}
$$

Two terms of output state are useful for the generation of entangled three photon as follows

$$
\frac{1}{2 \sqrt{2}}\left|1_{V}, 2_{H}\right\rangle_{3,4} \text { and } \frac{1}{2}\left|1_{H}, 1_{V} 1_{H}\right\rangle_{3,4}
$$

The states $\left|2_{H}\right\rangle_{4}$ and $\left|1_{V} 1_{H}\right\rangle_{4}$ are injected into the BS2 which the output state can be written as

$$
\left|2_{H}\right\rangle_{4}=\frac{1}{2}\left|2_{H}, 0\right\rangle_{5,6}+\frac{1}{\sqrt{2}}\left|1_{H}, 1_{H}\right\rangle_{5,6}+\frac{1}{2}\left|0,2_{H}\right\rangle_{5,6}
$$




$$
\begin{aligned}
\left|1_{V} 1_{H}\right\rangle_{4}= & \frac{1}{2}\left|1_{V} 1_{H}, 0\right\rangle_{5,6}+\frac{1}{2}\left|1_{V}, 1_{H}\right\rangle_{5,6}+\frac{1}{2}\left|1_{H}, 1_{V}\right\rangle_{5,6}+ \\
& \frac{1}{2}\left|0,1_{V} 1_{H}\right\rangle_{5,6}
\end{aligned}
$$

Consequently, the output state becomes the entangled three photon can be achieved by post selection as follows

$$
\left|1_{V}\right\rangle_{1}\left|1_{H} 1_{H}\right\rangle_{2}=\frac{1}{4}\left(|V H H\rangle_{356}+|H H V\rangle_{356}+|H V H\rangle_{356}\right)
$$

The success probability of generating three photon entangled is $3 / 16$ with a perfect post selection. The results of method will evolve as follows Eq.(4), Eq.(8) and Eq.(12).

\section{Conclusions}

This paper presented the scheme for generation of three entangled-photon without any non-linear optical process. The three entangled-photon can be achieved by post selection based on photon interferometry at the beam splitter. The success probability of generating three entangled-photon is $3 / 16$ and the method was compared with the previous method as shown in Table 1. This proposed method used only two beam splitters while another method used more elements for generation of three entangled-photon. However, the modified technique is depended on the theoretical of quantum mechanics for the generation of three entangled-photon which is the physical mechanism.

TABLE 1. COMPARISON OF THE SUCCESS PROBABILITY FOR PREPARING OF GENERATE TO THREE PHOTON ENTANGLED STATE.

\begin{tabular}{|c|c|c|}
\hline \multirow{2}{*}{ Paper } & \multicolumn{2}{|c|}{$\begin{array}{c}\text { The generation of three photon entangled state } \\
\text { with linear optical elements }\end{array}$} \\
\cline { 2 - 3 } & The photon entangled state & $\begin{array}{l}\text { Success } \\
\text { probability }\end{array}$ \\
\hline In [26] & $\frac{1}{4}\left(|H H V\rangle_{456}+|H V H\rangle_{456}+|V H H\rangle_{456}\right)$ & $3 / 16$ \\
\hline \multirow{2}{*}{ In [23] } & $\frac{1}{\sqrt{3}}\left(|H H V\rangle_{324}+|H V H\rangle_{324}+|V H H\rangle_{324}\right)$ & $2 / 27$ \\
\hline \multirow{2}{*}{ In [27] } & $\frac{-\sqrt{2}\left(|V H H+H V H+H H V\rangle_{567}+\right.}{8}\left(|V H H+H V H+H H V\rangle_{568}+\right.$ & \\
& $|V H H+H V H+H H V\rangle_{578}^{+}$ \\
& $\left.|V H H+H V H+H H V\rangle_{678}\right)$ & $3 / 8$ \\
\hline \multirow{2}{*}{$\begin{array}{c}\text { Proposed } \\
\text { method }\end{array}$} & $\frac{1}{4}\left(|V H H\rangle_{356}+|H V H\rangle_{356}+|H H V\rangle_{356}\right)$, & \\
& $\frac{1}{4}\left(|H V V\rangle_{356}+|V V H\rangle_{356}+|V H V\rangle_{356}\right)$, & \\
& $\frac{1}{4}\left(|V H H\rangle_{356}+|H H V\rangle_{356}+|H V H\rangle_{356}\right)$ & \\
\hline
\end{tabular}

\section{ACKNOWLedgment}

The authors would like to gratefully acknowledge Mr. Sujint Wangsuya, and Mr. Pituk Panthong for their kind help, valuable advice, supervision, and critical comments.

\section{REFERENCES}

[1] L. Mandel "Quantum effects in one-photon and two-photon interference" Rev. Mod. Phys. 71 (1999) S274.

[2] C. K. Hong, Z. Y. Ou, and L. "MandelMeasurement of subpicosecond time intervals between two photons by interference" Phys. Rev. Lett. 59 (1987) 2044.

[3] Y. H. Shih and C. O. Alley "New Type of Einstein-Podolsky-RosenBohm Experiment Using Pairs of Light Quanta Produced by Optical Parametric Down Conversion” Phys. Rev. Lett. 61 (1988) 2921.

[4] Z. Y. Ou and L. Mandel "Violation of Bell's inequality and classical probability in a two-photon correlation experiment" Phys. Rev. Lett. 61 (1988) 50.

[5] T. E. Kiess, Y. H. Shih, A. V. Sergienko, and C. O. Alley "EinsteinPodolsky-Rosen-Bohm experiment using pairs of light quanta produced by type-II parametric down-conversion" Phys. Rev.Lett. 71 (1993) 3893.

[6] Y. J. Lu, R. L. Campbell, and Z. Y. Ou, "Mode-locked two-photon states," Phys. Rev. Lett.91, 163602 (2003).

[7] M. A. Sagioro, C. Olindo, C. H. Monken, and S. Padua, "Time control of two-photon interference," Phys. Rev.A69, 053817 (2004).

[8] A. Zavatta, S. Viciani, and M. Bellini, "Recurrent fourth-order interference dips and peaks with a comblike two-photon entangled state," Phys. Rev. A70, 023806 (2004).

[9] C. K. Hong, Z. Y. Ou, and L. Mandel, "Measurement of subpicosecond time intervals between two photons byinterference," Phys. Rev. Lett.59, 2044-2046 (1987).

[10] P. G. Kwiat, K. Mattle, H. Weinfurter, A. Zeilinger, A. V. Sergienko, and Y. Shih, "New high-intensity source of polarization-entangled photon pairs" Phys. Rev. Lett. 75 (1995) 4337.

[11] P. G. Kwiat, E. Waks, A. G. White, I. Appelbaum, and P. H. Eberhard, "Ultrabright source of polarization entangled-photon" Phys. Rev. A 60 (1999) R773.

[12] P. G. Kwiat, P. H. Eberhard, A. M. Steinberg, and R. Y. Chiao, "Highefficiency single-photon detectors" Phys.Rev. A 49 (1994) 3209.

[13] D. Branning, W. Grice, R. Erdmann, and I. A. Walmsley "Interferometric technique for engineering indistinguishability and entanglement of photon pairs " Phys. Rev.A 62 (2000) 013814.

[14] Y.-H. Kim, M. V. Chekhova, S. P. Kulik, M. H. Rubin, and Y. Shih "High-intensity pulsed source of space-time and polarization doubleentangled photon pairs “, Phys. Rev. A 63 (2001) 062301.

[15] M. Fiorentino, G. Messin, C. E. Kuklewicz, F. N. C. Wong, and J. H.Shapiro "High-flux source of polarization-entangled photons from a periodically poled KTiOPO4 parametric down-converter" Phys. Rev. A 69 (2004) 041801.

[16] A. Yoshizawa and H. Tsuchida "Generation of polarization-entangled photon pairs at $1550 \mathrm{~nm}$ using two PPLN waveguides", Electron. Lett. 39 (2003) 621.

[17] A. Yoshizawa and H. Tsuchida "Violation of Bell's inequality in 1550 $\mathrm{nm}$ band without subtraction of accidental coincidences", Jpn. J. Appl. Phys. 44 (2005) L375.

[18] B.-S. Shi and A. Tomita "Generation of a pulsed polarization entangled photon pair using a Sagnac interferometer", Phys. Rev. A 69 (2004) 013803 .

[19] T. Kim, M. Fiorentino, and F. N. C. Wong "Phase-stable source of polarization-entangled photons using a polarization sagnac interferometer", Phys. Rev. A 73 (2006) 012316.

[20] Y.-H.Kim, S. P. Kulik, and Y. Shih, "Bell-state preparation using pulsed nondegenerate two-photon entanglement," Phys. Rev. A. 63, 060301(2001).

[21] J. Jacobson, G. Bjork, I. Chuang, and Y. Yamamoto, "Photonic de Broglie waves," Phys. Rev. Lett.74, 4835-4838(1995).

[22] K. Edamatsu, R. Shimizu, and T. Itoh, "Measurement of the Photonic de Broglie Wavelength of Entangled Photon Pairs Generated by 
Spontaneous Parametric Down-Conversion," Phys. Rev. Lett.89, 213601 (2002)

[23] XuBo Zou, K. Pahlke, and W. Mathis, "Generation of an entangled four-photon W state", Phys. Rev.A 66, 044302 , 2002

[24] Shi B S and Tomita A, "Creation of a polarization W state using optical fibre multiports”, J. Mod. Opt. 52, p.755-761, 2005

[25] Xia Y and Song H S, "Linear optical protocol for preparation of Nphoton Greenberger-Horne-Zeilinger state with conventional photon detectors", Appl. Phys. Lett. 92 021127, January 2008

[26] Tashima T, Ozdemir S K, Yamamoto T, Koashi M and Imoto N , "An elementary optical gate for expanding entanglement web", Phys. Rev. A 77 030302, March 2008

[27] Sheng Yu Bo, Deng Fu Guo, Zhou Hong Yu, "Generation of Multiphoton Entangled States with Linear Optical Elements", Chin. Phys. Lett. 25 3558, July 2008

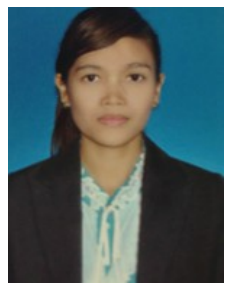

Siriporn Saiburee received B.S. in Science, majoringin Physics from Naresuan university, in 2006. She is currently pursuing a master degree in Electrical and Computer Engineering at Thammasat university, Thailand. Her research interest includes quantum optics, optoelectronics and linear optical.

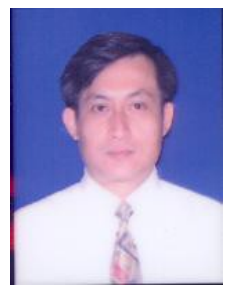

Wanchai Pijitrojana received B.S. In Telecommunication from King Mongkut's Institute of Technology Ladkrabang, M.S. in Computer Technogy from Asian Institute of Technology , M.S. in Nonlinear Optics from University of Southern California, California, USA and Ph.D. in Optoelectronics from King's College, University of London, London, UK respectively. His research interest includes optoelectronics, quantum optics and nonlinear optics. 